\title{
FORÇA MUSCULAR RESPIRATÓRIA, PICO DE FLUXO E INDICE DE MASSA CORPÓREA EM INDIVÍDUOS ADULTOS COM SINDROME DE DOWN
}

Elaine Aparecida Lozano da Silva, Giulia Iracelis Passarini da Silva, Ana Paula Costa Monteiro, Renilton José Pizzol

Curso de Fisioterapia da Universidade Estadual Paulista Júlio de Mesquita Filho - Faculdade de Ciências e Tecnologia FCT/UNESP. E-mail: elaine 1060@hotmail.com

\section{RESUMO}

Indivíduos com síndrome de Down (SD) apresentam predisposição á infecções respiratórias, considerada principal causa de hospitalização e mortalidade. O objetivo deste estudo foi mensurar os valores do pico de fluxo expiratório, cálculo de índice de massa corpórea (IMC) e avaliar a força muscular respiratória de 19 indivíduos (20 á 51 anos) com SD. A avaliação foi realizada segundo protocolos pré-estabelecidos para obtenção de peso e altura, força muscular respiratória (abdominal, intercostais e diafragma) e do pico de fluxo expiratório. Sendo assim a média do IMC foi de $31,9 \pm 6 \mathrm{~kg} \backslash \mathrm{m}^{2}$, prevaleceu nesta população força muscular regular em $73,7 \%$ dos indivíduos no músculo abdominal, $42,1 \%$ diafragma e $36,8 \%$ intercostais e o pico de fluxo expiratório de 148,8 \pm 50,3 L \اmin. Constatando-se que os indivíduos com SD apresentaram uma insatisfatória força muscular respiratória, valores baixos de pico de fluxo expiratório e índices de sobrepeso/obesidade revelando a presença de fatores predisponentes para problemas respiratórios.

Palavras-chave: Síndrome de Down, Avaliação fisioterapêutica respiratória; Pico de fluxo expiratório; Força muscular respiratória; Índice de massa corpórea.

\section{INTRODUÇÃO}

A síndrome de Down (SD) se caracteriza, em sua etiologia, por apresentar alteração na divisão cromossômica, resultando na triplicação do material genético referente ao cromossomo 21, sendo considerada a principal causa de deficiência intelectual (POLICARPO, SANTOS, 2011).

De acordo com o IBGE (2010) o número de pessoas com SD no Brasil é de cerca de 270 mil pessoas, sendo a proporção de um para cada 700 nascidos vivos tendo como principal fator de risco a idade materna, principalmente em mães com idade acima de 35 anos (GUSMÃO, TAVARES, MOREIRA, 2003).

Segundo Romano (2007) e Castoldi, Périco, Grave (2012) além de graus variáveis de deficiência intelectual, a SD está associada à vários problemas de saúde entre os quais problemas físicos gerais, transtornos mentais, alterações osteomusculares e doenças cardíacas, neurológicas, oftalmológicas e gastrointestinais. Outros importantes problemas se encontram no sistema respiratório, pois o individuo com SD apresenta várias anormalidades anatômicas e fisiológicas 
que o predispõe ao aparecimento de complicações como infecções respiratórias recorrentes, que são consideradas a principais causas de hospitalização e mortalidade na SD (SOARES et al., 2004).

Dentre as várias alterações respiratórias encontra-se a fraqueza muscular respiratória que é causada pela hipotonia generalizada que ocorre nesses indivíduos que leva a um quadro de flacidez muscular e ligamentar (SCHUSTER, ROSA, FERREIRA, 2012). Segundo Silva \& Kleinhans (2006) a hipotonia muscular é considerada grande responsável pelos vários problemas respiratórios desenvolvidos em indivíduos com SD estando relacionada ao enfraquecimento do músculo diafragma, à baixa responsividade dos reflexos de tosse, à diminuição da capacidade de expectoração e à alterações posturais importantes. Além disso, segundo Silva (2008) a hipotonia altera a configuração da caixa torácica, reduzindo ainda mais a força muscular respiratória, facilitando o aparecimento de infecções respiratórias.

Outro aspecto importante para o mau funcionamento do sistema respiratório nos indivíduos com SD é o excesso de peso. Segundo Zini e Ricalde (2009) esse indivíduos apresentam predisposição para o excesso de peso e obesidade, podendo este ser decorrente da redução na taxa metabólica basal, de maus hábitos alimentares e do déficit de crescimento.

Para Silva (2008) pessoas obesas podem apresentar alterações no sistema respiratório que ocasionam a redução do volume de reserva expiratória e provocam uma resistência ao fluxo aéreo, podendo estes ser causados devido à redução na elasticidade pulmonar e à elevação do músculo diafragma colocando-o em posição de desvantagem mecânica e em situação de gerar menos força o que torna a avaliação do peso corpóreo nessas pessoas uma questão importante na detecção de problemas relacionados às alterações respiratórias que podem reforçar o mau funcionamento pulmonar.

Nesse sentido a avaliação fisioterapêutica respiratória em indivíduos com SD por meio de metodologias que pertencem ao cotidiano clínico do fisioterapeuta pode possibilitar a investigação da situação muscular respiratória e do peso corpóreo que pode ser de fundamental importância para detectar precocemente possíveis problemas e evitar as complicações respiratórias sempre indesejáveis para esses indivíduos e contribuir para aumentar a sua expectativa de vida e sua qualidade de vida que, segundo Meyer et al (2002) e Marques, Nahas (2003) deveriam ser aspectos cada vez mais presentes nas ações em saúde voltadas para os indivíduos com SD.

Posto isso, o objetivo deste estudo é avaliar a força muscular respiratória, o pico de fluxo expiratório e o peso corpóreo de indivíduos com SD. 


\section{METODOLOGIA}

Realizou-se um estudo de caráter transversal descritivo com indivíduos adultos com SD, atendidos na Associação de Pais e Amigos dos Excepcionais (APAE) de Presidente Prudente-SP.

O procedimento metodológico foi desenvolvido a partir das seguintes etapas:

a) Elaboração de uma ficha contendo um cabeçalho com a identificação dos dados pessoais do individuo participante (nome, sexo, idade e data de nascimento); uma ficha de avaliação física contendo itens sobre dados: antropométricos (peso e altura) que foram usados para o calculo do Índice de Massa Corporal (IMC); da força muscular respiratória e do pico de fluxo expiratório.

b) Realização da avaliação respiratória segundo protocolos de avaliação pré-estabelecidos. Para a avaliação do peso e para a medida da estatura foi seguido o protocolo sugerido por Júnior et al (2009). Já para a avaliação muscular o método empregado foi o da palpação muscular que foi realizado segundo o preconizado por Costa (2002) conforme os quadros 1 e 2.

Quadro 1. Avaliação dos músculos diafragma e intercostais por meio da palpação.

\begin{tabular}{|l|l|}
\hline Diafragma & Intercostais \\
\hline $\begin{array}{l}\text { Bom - abdome da pessoa expulsa mão do } \\
\text { examinador }\end{array}$ & $\begin{array}{l}\text { Bom - perceptível aumento dos espaços } \\
\text { intercostais e horizontalização das costelas }\end{array}$ \\
\hline $\begin{array}{l}\text { Regular - tentativa de expulsão da mão do } \\
\text { examinador com expansão do tórax em vez do } \\
\text { abdome }\end{array}$ & $\begin{array}{l}\text { Regular - discreto aumento dos espaços } \\
\text { intercostais e costelas permanecem } \\
\text { verticalizadas }\end{array}$ \\
\hline $\begin{array}{l}\text { Ruim - percebe-se contração, mas não há } \\
\text { expulsão da mão do examinador }\end{array}$ & $\begin{array}{l}\text { Ruim - espaços intercostais permanecem } \\
\text { imóveis e costelas verticalizadas }\end{array}$ \\
\hline $\begin{array}{l}\text { Traço - não há expulsão da mão do examinador } \\
\text { nem se sente qualquer contração }\end{array}$ & $\begin{array}{l}\text { Traço - idem ao ruim, porém sem percepção } \\
\text { de contração muscular }\end{array}$ \\
\hline
\end{tabular}

Fonte: Costa (2002)

Quadro 2. Classificação da força dos músculos abdominais.

Bom - flexão total do tronco

Regular - flexão parcial do tronco, com elevação da cabeça, ombros e escápulas

Ruim - flexão da coluna cervical

Traço - leve contração do abdome anterior quando a pessoa tenta tossir

Fonte: Costa (2002)

c) Para a realização da avaliação do pico de fluxo expiratório foi utilizado um aparelho Peak Flow da marca ASSES, com variação de 60 a $800 \mathrm{l} / \mathrm{min}$. O protocolo do teste foi realizado de acordo com o preconizado por Parreira et al (2007): o indivíduo foi orientado a sentar com a coluna apoiada no encosto da cadeira, joelhos a $90^{\circ}$, pés apoiados no chão, mãos segurando o 
bocal e utilização de um clipe nasal na realização da manobra, e orientado a realizar a partir de uma inspiração máxima, uma expiração forçada máxima, curta e explosiva. Para melhorar o entendimento foi utilizado o protocolo de Santiago et al (2008), durante a realização do esforço expiratório com a frase "Assopre uma vela o mais rápido possivel".

Para maior reprodutibilidade da mensuração foram realizadas três medidas (com um intervalo de repouso de 30 - 40 segundos entre cada manobra), sendo que a manobra foi repetida até que três registros estivesse dentro de $20 \mathrm{~L} / \mathrm{min}$ cada um do outro. Para efeito de análise foi considerada a medida de maior valor (PARREIRA et al, 2007).

A avaliação foi iniciada somente após a assinatura dos responsáveis pelos indivíduos participantes da pesquisa do termo de consentimento livre e esclarecido. Este estudo foi aprovado pelo Comitê de Ética Local da Faculdade de Ciências e Tecnologia da UNESP com o no 09655413.5.0000.5402/2013.

\section{RESULTADOS}

A população deste estudo foi constituída por 19 pessoas adultas com SD sendo 10 mulheres e nove homens, com média de idade de 31,9 \pm 9 anos, de peso de 73,26 $\pm 14,84 \mathrm{~kg}$, de altura de 1,49 $\pm 0,05$ metros e de IMC de 31,9 $\pm 6 \mathrm{~kg} \backslash \mathrm{m}^{2}$.

Os resultados obtidos mostraram que para a força muscular diafragmática, $42,1 \%$ dos indivíduos avaliados apresentaram boa força, 42,1\% força regular, 5,2\% força ruim e 10,5\% não conseguiram realizar o teste. Já para a força muscular dos intercostais $31,6 \%$ dos indivíduos apresentaram boa força, 36,8\% força regular, $21,0 \%$ força ruim e 10,5\% não conseguiram realizar o teste. Quanto à força muscular abdominal 5,2\% dos indivíduos apresentaram boa força, 73,7 \% força regular, 10,5 \% força ruim e 10,5\% dos indivíduos não conseguiram realizar o teste.

Quanto aos valores do pico de fluxo expiratório mensurado o valor médio encontrado para os homens foi de $186,7 \pm 39,2 \mathrm{~L} / \mathrm{min}$ e para as mulheres de $151,5 \pm 60,8 \mathrm{~L} / \mathrm{min}$.

\section{DISCUSSÃO}

De acordo com os valores obtidos no presente trabalho, no que diz respeito à força muscular, observou-se que a maioria dos indivíduos avaliados apresentou valores insatisfatórios de contração muscular, principalmente no caso dos músculos abdominais revelando que a possível hipotonia muscular característica desses indivíduos tenha interferido na perda de força muscular respiratória o que já era esperado. 
Em relação aos valores do pico de fluxo expiratório foram observados baixos valores tanto para o sexo feminino quanto para o masculino quando comparados com os valores obtidos de uma população adulta sem SD. Em relação ao estudo de Romano (2007), por exemplo, cujos valores encontrados para indivíduos com idade ente 20 à 50 anos e altura média de 1,50 m foi de $529,6 \mathrm{~L} / \mathrm{min}$ para homens e de 401,7 L/min para mulheres, neste estudo os indivíduos avaliados apresentaram um pico de fluxo expiratório de apenas 35,2\% do obtido na população masculina e de $37,7 \%$ na população feminina. Esses resultados podem ser explicados pela insatisfatória força muscular observada já que o pico de fluxo expiratório também depende também da força muscular expiratória revelando uma possível repercussão da hipotonia sobre o funcionamento pulmonar.

Já quanto ao IMC foi observado que a população avaliada apresentou no geral valores de obesidade sendo que os indivíduos do sexo feminino apresentaram um IMC de $32,6 \mathrm{~kg}$ e os do sexo masculino de $31,36 \mathrm{~kg}$ revelando um IMC mais elevado nas mulheres o que também foi observado no estudo realizado por Silva et al (2006) com indivíduos adultos com SD que observaram excesso de peso e obesidade em $68,5 \%$ dos homens e $82,3 \%$ no sexo feminino. Neste estudo o percentual de sobrepeso e obesidade também foi bastante alto com $21 \%$ dos indivíduos apresentando sobrepeso e $54,3 \%$ sendo classificados como obesos.

\section{CONCLUSÃO}

O presente estudo mostrou que indivíduos com SD apresentaram uma insatisfatória força muscular respiratória, valores muito baixos de pico de fluxo expiratório e índices de sobrepeso/obesidade revelando a presença de fatores predisponentes para problemas respiratórios indicando a necessidade da avaliação fisioterapêutica respiratória para esse tipo de população.

\section{REFERÊNCIA}

CASTOLDI, A.; PÉRICO, E.; GRAVE, M. Avaliação da força muscular e capacidade respiratórias em pacientes com síndrome de down após Bad Ragaz. Rev Neurocienc, v. 20, n. 3, p. 386-391, 2012.

COSTA, D. Fisioterapia Respiratória Básica. São Paulo: Atheneu, 2002. p. 127.

GUSMÃO, F. A. F.; TAVARES, E. J. M.; MOREIRA, L. M. A. Idade materna e Síndrome de Down no Nordeste do Brasil. Cad. Saúde Pública, Rio de Janeiro, v. 19, n. 4, p. 973-978, jul.-ago., 2003. 
JUNIOR, I. F. F.; et al. Padronização de técnicas antropométricas. 1. ed. São Paulo: Cultura Acadêmica: Universidade Estadual Paulista, Pró - Reitoria de Graduação, p. 70, 2009.

MARQUES, A. C.; NAHAS, M. V. Qualidade de vida de pessoas portadoras de Síndrome de Down, com mais de 40 anos, no Estado de Santa Catarina. R. Bras. Ci. e Mov, Brasília, v. 11, n. 2, p. 55-61, junho, 2003.

MAYER, A. F.; et al. Fisioterapia Respiratória. In: TARANTINO, A. B. Doenças Pulmonares. Rio de Janeiro: Guanabara Koogan, p. 536-548, 2002.

PARREIRA, V. F.; et al. Pressões expiratórias máximas: valores encontrados e e preditos em indivíduos saudáveis. Rev. bras. fisioter., São Carlos, v. 11, n. 5, p. 361-368, set./out., 2007.

POLICARPO, C. L.; SANTOS, L. J. Avaliação da Força Muscular Respiratória em Indivíduos Portadores de Síndrome de Down. Rev. Inspirar movimento \& saúde, v. 3, n. 2, p. 26-30, Março/abril, 2011.

ROMANO, A. M. C. Avaliação funcional respiratória em indivíduos com Síndrome de Down. 2007. p. 78 . Dissertação (Mestrado em Intervenção Fisioterapêutica.) - Universidade Metodista de Piracicaba, Piracicaba.

SANTIAGO, S. Q.; et al. Avaliação da força muscular respiratória em crianças e adolescentes com sobrepeso/obesos. Rev. Paul. Pediatr, v. 28, n. 2, p. 146-50, março, 2008.

SCHUSTER, R. C.; ROSA, L. R.; FERREIRA, D. G. Efeitos do treinamento muscular respiratório em paciente portadores de síndrome de Down: estudo de casos. Rev Fisioter S Fun, Fortaleza, v.1, n.1, p. 52-57, 2012.

SILVA, D. L.; et al. Avaliação da Composição Corporal em Adultos com Síndrome de Down. Arquivos de medicina, v. 20, n. 4, 2006.

SILVA, M. F. M. C.; KLEINHANS, A. C. S. Processos cognitivos e plasticidade cerebral na Síndrome de Down. Rev. Bras, Marilia, v. 12, n.1, p. 123-138, Jan.-Abr. 2006.

SILVA, V. Z. Avaliação da função pulmonar e da força muscular respiratória em indivíduos com retardo mental, portadores e não portadores de Trissomia 21 no Distrito Federal. Dissertação de Mestrado em Universidade de Brasília, 2008.

SOARES, J. A.; et al. Distúrbios respiratórios em crianças com síndrome de Down. Arq Ciênc Saúde, São José do Rio Preto, v. 11, n. 4, p. 230-3, out.-dez. 2004.

ZINI, B.; RICALDE, S. R. Características nutricionais das crianças e adolescentes portadoras de síndrome de Down da APAE de Caxias do Sul e São Marcos - RS. Rev. Pediatria, São Paulo, v. 31,n. 4, p. 252-9, 2009. 